\title{
A laser-based technique for measuring accuracy and distortion in judgments of linear dimensions
}

\author{
THOMAS R. ALLEY \\ Clemson University, Clemson, South Carolina
}

\begin{abstract}
A new technique is described that permits precise measurement of accuracy and distortion in judgments of linear dimensions based on either perception or memory. This technique involves the use of a single laser beam and a reference line placed on a projection surface. By rotating a laser device, the distance between the reference line and the point created by the beam may be continuously varied. This procedure avoids unintentional distortion from misjudgment of standard metrics, while the semicircular movement required by this technique eliminates body-referenced estimation and some other potential confounds. Potential applications to research in visual perception, spatial memory, and body image are discussed.
\end{abstract}

A concern for estimates of linear extent (e.g., hip width, vehicle width or height, bolt length) arises in many contexts, including basic research in perception, clinical studies (e.g., judgments of body image), and industry. This article describes a new technique that uses a single laser device to obtain precise measurement of accuracy and distortion in judgments of linear dimensions. This new technique utilizes observer-controlled projection of a laser beam, a procedure that is similar to previous use of a laser pointer for measurement of perceived eye height (e.g., Matin \& Li, 1995). More specifically, the new technique involves the use of a single beam emanating from a laser attached to a swivel and aimed toward a reference line. By rotating the laser device, the distance between the reference line and the point created by the beam may be continuously and accurately varied. This article presents some of the inherent advantages of this method, as compared with alternative techniques, and describes the construction and use of such laser devices. Potential applications in the areas of visual perception, spatial memory, and body image assessment also are presented. Also discussed is a second new technique that involves the use of adjustable belts and is particularly suitable for obtaining threedimensional estimates of body size, such as waist size.

Several techniques have been used to collect estimates of linear extent, but all have significant weaknesses or limitations. Perhaps the most obvious and common approach is to ask for estimates in standard units of measurement (e.g., inches or meters). The main problem with this ap-

Portions of this article were presented at the Tenth International Conference on Perception and Action (ICPA-X), held August 1999 at the University of Edinburgh, Scotland. Thanks to C. Brennan for serving as the experimenter and for additional help with data collection. I also thank J. Vaughan and an unidentified reviewer for helpful comments on an earlier version of this manuscript. Correspondence concerning this article should be addressed to T. R. Alley, Department of Psychology, 418 Brockett Hall. Clemson University, Clemson, South Carolina 29634-1355 (e-mail: alley (aclemson.edu). proach is that people will often have distorted perceptions of these units. Consequently, it is likely that very few people can precisely apply standard measurement units without the aid of a measurement device, such as a tape measure or a yardstick. So if asked to judge, say, the width of a room in meters, even people who accurately perceive the width will overestimate it if their mental representation of the length of a meter is too short. Furthermore, responses in standard units are likely to suffer from imprecision, owing to rounding and quantizing errors (e.g., reporting in units divisible by 5 or 10 ). The often misleading picture of perception presented by numerical estimates is revealed by the higher accuracy of the corresponding estimates implied or exhibited in action tasks (e.g., accuracy in walking blindfolded to a previously seen location; Cutting \& Vishton, 1995).

A second major problem with this technique of verbal estimation is that some estimates may be based more on remembered dimensions than on perceived or remembered size. That is, estimates may be aided or distorted by users' memories of specific linear dimensions, such as remembering that the ceiling height is $8 \mathrm{ft}$ or knowing that you have a 30 -in. television set. Likewise, when people are asked for a series of verbal estimates, their memory of previously generated responses may influence subsequent responses.

Other measurement techniques also may be influenced by two heuristics that are less obvious than reliance on memory of dimensions. These are body-referenced judgments (e.g., knowing that something is about shoulder wide) and motoric memories (e.g., replicating the movement needed to place a hand on each side of an object). By moving the site of measurement away from the body (to the projection surface), the laser technique should minimize the influence of body-referenced judgments. By requiring an unfamiliar rotary (semicircular) movement wherein small changes in position produce much larger changes in indicated distance, the laser technique eliminates the usefulness of motoric memories. 
It should be noted that body-referenced estimations provide a particularly useful approach for some questions about judgments of linear extent. Such estimates are central to an affordance-based approach, advocated by Warren and others (see Warren \& Whang, 1987). This approach takes advantage of the body-referenced or action-based judgments that are critical in everyday life, such as when deciding where to place one's feet when traversing a rough terrain. Limitations of this approach include its suitability for judgments of size only at or near those of various affordances, such as the "passability" of an opening (Warren \& Whang, 1987) or the crossability of a gap (Jiang \& Mark, 1994; Mark, Jiang, King, \& Paasche, 1999). A related problem is that of precision: Precise judgments can only be expected when the to-be-judged size has an exact counterpart in body size or movement (e.g., shoulder width or maximum stride length, but probably not lengths that are, say, double these).

A variety of simple methods that can be useful for assessing many linear judgments involve reproducing the distance. This can be done by marking on a writing surface (the marker method; e.g., Askevold, 1975), moving marker objects (e.g., Gilinsky, 1955), or manipulating an apparatus that creates variable indicators of linear extent (Ruff \& Barrios, 1986; Thompson \& Spana, 1988). Depending on how it is implemented, these reproduction techniques may be limited to a maximum feasible length of a few meters, at most, and may require a somewhat complicated, custom-built apparatus. Furthermore, most reproduction techniques allow people making judgments to use body parts as guides, something that may not be desirable. For instance, a study investigating the accuracy of memory for length of one's hand should not use the marking method. Moreover, body-referenced judgments may distort the accuracy with which linear extent can be judged while operating machinery or in many natural contexts. The semicircular movement required with the new technique described below eliminates body-referenced estimation, as well as the distortion due to misjudgment of standard metrics, yet allows precise estimates over a wide range of linear extents.

\section{MATERIALS AND CONSTRUCTION}

The main piece of equipment is a laser pointer or similar device. Ideally, this device should generate a very narrow beam producing a small round point at a distance of up to $5 \mathrm{~m}$. Researchers are cautioned that many inexpensive laser pointers create a spot of light that will be over $1 \mathrm{~cm}$ in diameter at a distance of $10 \mathrm{~m}$ and that may be irregularly shaped. Most such lasers should still be usable but will require researchers to instruct their subjects that the center of the beam is to be used. Lasers also vary in power, but all common low-powered lasers should create a beam of adequate intensity in laboratory settings. If used at very long distances or in brightly lit settings, such as in certain outdoor situations during daylight, a more powerful (at least 4-mW) laser may be necessary.

The laser device should be mounted on a swivel or a pivot to allow free rotary movement in just one plane. Appropriate swivels are available at many hardware stores, where they are often sold for use in kitchen cabinets. To enhance users' control over the direction of the beam, a rod or handle may be attached to the laser device and used to manipulate its rotary motion. The sensitivity to motion will increase with greater distance to the projective surface but will be an inverse function of rod length.

Most lasers pose a hazard in the form of potential retinal damage if directed into an eye. For this reason, it is especially important that the laser device be securely attached and that all present during its use understand this danger. Furthermore, it would be prudent to mount the laser so that it is always projected well below the eye height of whoever is responsible for marking or measuring beam locations. The low-powered laser pointing devices that are likely to be used because they are inexpensive and widely available pose little risk when used prudently.

\section{PROCEDURE}

A clearly visible reference line or point should be placed at a suitable distance from the observer and laser apparatus. The use of the device is quite simple, but to prevent any confusion, all users should be explicitly told that the distance between the reference line or point and the center of the laser beam's point will be recorded as their estimate of linear extent. Observers should be shown, or allowed to explore, the changing position of the laser beam as the attached handle is manipulated. Practice trials should not be necessary, since the observers can quickly and easily adjust their indicated lengths in either direction until satisfied.

By mounting the laser device on a tripod, users may estimate linear extent along any axis in a plane. In this case, a small but easily detected reference point is needed, rather than a reference line.

To record the estimates, the experimenter may mark the spots on the projection surface targeted by the user for later measurement or take measurements immediately as they are made. In certain cases (see below), it may be useful to clearly mark some judgments, so that they may serve as reference points for the user as he or she makes additional estimates. That is, the previously marked responses can be used to guide later judgments that the observer may think of as relative (e.g., greater than or less than) judgments. For this purpose, a marker board makes a good projection surface.

\section{USABILITY AND RELIABILITY}

To test the usability and reliability of this method, a study was conducted in which judgments of the size of 


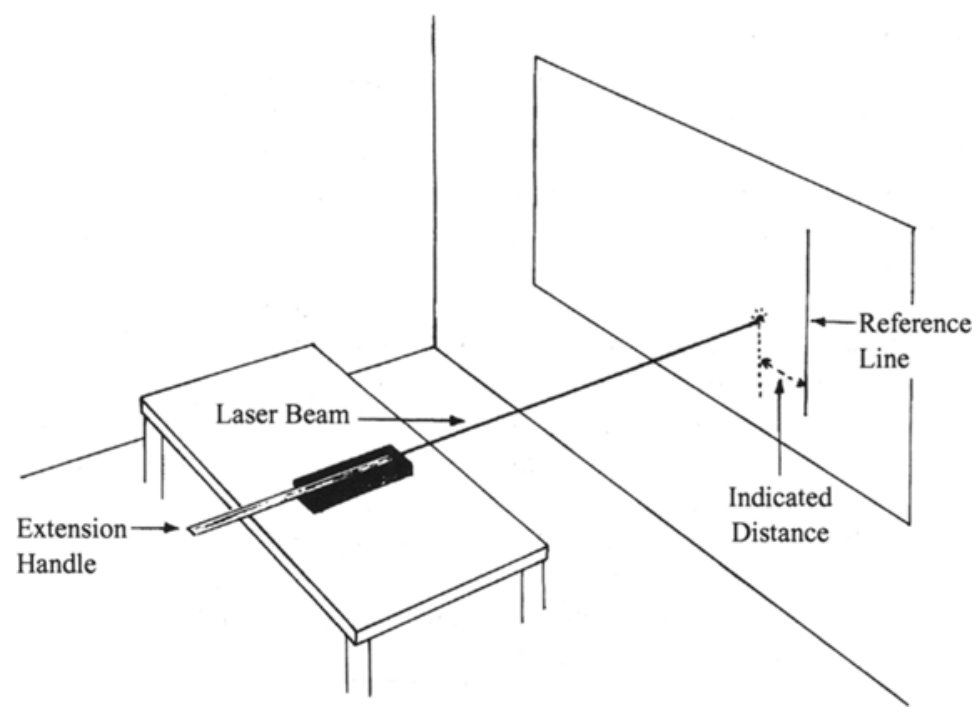

Figure 1. Diagram showing setup of laser apparatus, including the extension handle used to control the laser beam and a vertical reference line. Not apparent is the swivel mount for the laser device.

several objects, of another person, and of the participant's own body were made on two separate occasions 1 week apart.

\section{Method}

Participants. The participants were 32 students, including 13 females and 19 males. (Two additional participants had to be eliminated when they failed to return for the second testing session.) Their age ranged from 18 to 24 years, with an average age of 19.13 years. All participants were volunteers from introductory psychology classes, who received extra credit for participating.

Materials. The participants used a light beam apparatus, as illustrated in Figure 1, to estimate the width of four objects, of a person, and of themselves. The four objects that the participants were to estimate were a black cabinet $(775 \mathrm{~mm})$, a green 12-in. $(350-\mathrm{mm})$ ruler, a curved-back chair with a rubber band indicating a position on the back that was $\mathbf{4 7 4} \mathrm{mm}$ wide, and a tapered cylinder (trashcan) marked with a rubber band at a position $295 \mathrm{~mm}$ wide. In addition, four plain black web belts varying only in length were prepared for use as an alternative measure of waist size. (These web belts were being tested for use as a highly sensitive measure of three-dimensional body image, not as a standard of comparison for the two-dimensional estimates obtained with the laser apparatus.) An anthropometric caliper (Carolina Biological Supply item 696245 ) was used to measure body area (with the distance measured with a meter stick, rather than with the somewhat inaccurate scale included on the caliper itself).

Procedure. The experimenter read instructions that included a reminder that this was a two-part study for which the participant would have to return exactly 1 week later. The participant was then told that he or she was going to be estimating the width of several objects, of a person, and of himself/herself. Next, the experimenter turned on the laser apparatus and explained how it was to be used (by moving the handle sideways).

Each participant began the experiment by using the laser beam to estimate the width of the objects. They stood about $3.5 \mathrm{~m}$ from a large white marker board with a red line and about $4.5 \mathrm{~m}$ from the objects being estimated. The cabinet, chair, 12 -in. ruler, and trashcan were presented in random order. The light beam always started at the red reference line. After the participant had estimated the width of an object by moving the light beam, the experimenter made a tiny, color-coded mark at the indicated spot on the board, then asked the participant to return the beam to a position near the reference line. Once the widths of all four objects were estimated, the experimenter measured and recorded the estimates marked on the board. When that was finished, the marks were erased, and the light beam returned to the red line.

The next task required the participant to estimate the width of the female experimenter's shoulders $(438 \mathrm{~mm})$, waist $(268 \mathrm{~mm})$, and hips $(346 \mathrm{~mm})$. The experimenter stood at the side of the room, about $3 \mathrm{~m}$ from the participant. Shoulders, waist, and hips were judged in random order, with the estimates being marked, measured, and then erased, as before.

The third task required the participant to estimate the width of his or her own waist, shoulders, and hips, without examining himself or herself. These estimates were made in a random order, with the estimates being marked, measured, and then erased, as before.

Finally, the participant was given a web belt and asked to adjust the belt (by making it bigger or smaller) to his or her own waist size. The participant was not allowed to put the belt on or hold it up to himself or herself. Next, the participant was asked to put on a different belt (identical except for length, which was varied to prevent the participant from using the length of the unused portion of the belt as an index to his or her previous estimate). The participant was to position and adjust this belt until he or she felt it was properly adjusted and at the same body position as that used in the immediately preceding estimate. After completing this task, the participant was dismissed, and the experimenter measured the used lengths of both belts.

When the participants came back 1 week later, the whole procedure was repeated, although new random orders of objects and body parts were used. In addition, after completing the belt task, the participants' hips, waist, and shoulders were measured, using body calipers.

\section{Results and Discussion}

None of the 32 participants displayed or expressed difficulty in using the laser to indicate perceived size. To assess test-retest reliability, Pearson correlations were computed for paired estimates of objects (4), experimenter's 
Table 1

Mean Pearson Correlation Coefficients for Repeated Estimates and Mean Percent Error, by Target and Target Category

\begin{tabular}{lllr}
\hline \multirow{1}{c}{ Target } & \multicolumn{3}{c}{ Test-Retest } \\
\cline { 3 - 4 } \cline { 3 - 4 } Actual Size (mm) & Reliability & Mean \% Error \\
\hline Inanimate objects & & & \\
Tapered cylinder & 295 & .52 & 18.80 \\
Cabinet & 775 & .45 & 15.72 \\
Chair back & 474 & .40 & -0.01 \\
Ruler & 350 & .73 & -10.98 \\
$\quad M$ & 473.5 & .53 & 5.88 \\
Female body & & & \\
Female's shoulders & 438 & .67 & -0.67 \\
Female's waist & 268 & .56 & 14.98 \\
Female's hips & 346 & .54 & 20.75 \\
$\quad M$ & 350.7 & .59 & 6.51 \\
Own body & & & \\
Own shoulders (mean) & 439 & .77 & 8.60 \\
Own waist (mean) & 271 & .69 & 35.75 \\
Own hips (mean) & 329 & .71 & 21.68 \\
$\quad M$ & 346.6 & .72 & 22.01 \\
Overall & 398.8 & .60 & 10.91 \\
\hline
\end{tabular}

body (3), and own body (3). (See Table 1.) All 10 correlation coefficients, ranging from .40 to .77 , were statistically significant $(p<.025)$, indicating moderate to high reliability. Very high reliability was found for the belt task $(r=.93)$, which, of course, most resembled an everyday activity. Perhaps owing to familiarity, the average correlation was higher for estimates of one's own body $(r=.72)$ than for estimates of the inanimate objects $(r=.53)$ or of the experimenter's body $(r=.59)$. The only significant differences between the two estimates occurred for three (of four) inanimate objects $[t(31)>2.65, p<$ $.02]$. The correlations between the accuracy (measured as percentage of error, see below) of each pair of estimates were somewhat higher: For the three target types, these ranged from .63 for inanimate objects to .64 for the female body to .75 for one's own body $(p<.001)$. These correlations should not be confused with the correlations between actual and estimated sizes, which ranged from .652 for estimates of one's own body to .945 for inanimate objects. Overall (i.e., across both objects and raters), there was a high correlation between actual and estimated sizes $(r=.90)$, which supports the validity of this technique for measurements of perceived size.

To assess and compare accuracy, the differences between actual and average estimated sizes were computed, then converted to percentage of errors to control for target size (see Table 1). Researchers should note that previous research using a variety of other techniques often revealed a tendency for observers to overestimate linear extent, particularly when judgments involved objects at a distance (Sedgwick, 1986). Thus, it is not surprising that there was a tendency for observers to overestimate linear extent. On average, the participants overestimated the size of their own body by $22 \%$. (This does not include es- timation using the web belts, for which the participants' average amount of overestimation was $52.3 \mathrm{~mm}$, or $5.7 \%$.) Highlighting this tendency, the mean estimate of waist size was higher than the actual width for all 32 participants! The size of the female experimenter and of the inanimate objects also tended to be overestimated, with overall average errors of $6.5 \%$ and $5.9 \%$, respectively. It is noteworthy that the participants were quite accurate overall for the female's shoulders, with much lower levels of accuracy for her waist and hips, which may be considered to be somewhat "disguised" by clothing.

The average percentages of errors for each of the three types of task were all significantly correlated $(r \geq .60$, $p<.001$ ), indicating that some people were consistently better than others at size estimation. Comparing the average percentage of error scores revealed significantly less accuracy overall for estimates of one's own body than for those of another person's body $[t(31)=10.03, p<$ $.001]$. Judgments of another person's body were not significantly less accurate than estimates for inanimate objects $[t(31)=0.33, p>.74]$. Although this makes it appears that estimation of one's own body size may be particularly subject to distortion, the data obtained with the web belts indicates otherwise. Specifically, circumference estimates made with the web belts were significantly more accurate than the estimates of waist width $[t(31)=8.98, p<.001]$ and roughly equal in accuracy to those of inanimate objects. Moreover, the fact that only estimates of one's own body were to be done from memory means that additional research with comparable viewing conditions, perhaps making use of a mirror, is needed to provide a fair test.

\section{GENERAL DISCUSSION}

In summary, laser-based assessment of perceived or remembered linear extent has high precision and appears to have good reliability. This method is suitable for a wide range of applications and should be a particularly good choice when it is desirable to avoid body-referenced judgments and those based on motoric memories. Depending on whether or not the objects of judgment are concurrently present, these judgments may be based on either perception or memory. Although this technique does not have sufficient precision to be used for very small (say, $1 \mathrm{~cm}$ or less) dimensions and is not feasible for measurement of large (over $40 \mathrm{~m}$ ) distances, it will work well for most judgments of linear dimensions in the intermediate range. Researchers should note that less handle movement is needed for a given amount of beam movement as distance from the reference line increases. This means that precise control of the laser beam will become increasingly difficult as distance increases for large targets, a problem that may be reduced by increasing the distance between the laser and the projection surface, thereby reducing the angle of projection necessary for a 
given distance. In addition, it is possible that this could produce systematic changes in accuracy for widely variant distances as target distance increases.

The following three sample areas of application illustrate a few potential uses for this flexible technique.

\section{Basic Research in Visual Perception}

This technique will provide an easy means of measuring the perceived length or distance in countless varieties of perceptual judgment. This includes not only judgments of the linear size of everyday objects or of the distance between them, but also visual illusions. For instance, indications of distance through use of this technique could be used to measure the strength of perceptual illusions of size, such as the Müller-Lyer or horizontal-vertical illusions (see Schiffman, 1996). To directly compare individuals, several standard objects may be presented to participants who are required to manipulate the laserpointing device to reproduce the specific linear dimensions of these objects.

\section{Measurement of Spatial Memory}

How wide is your car? What is the largest box that will fit through the front door? How wide is the viewable portion of your TV? Estimates to questions such as these can be easily collected with the laser-based technique, then compared with actual measurements, to reveal any systematic distortion in spatial memory.

To create a standard measure of accuracy for the purpose of comparing individuals, the experimenter simply needs to provide controlled exposure to a fixed sample of objects (e.g., a few boxes and cylinders) and, either immediately or after a uniform retention interval, measure judgments of linear dimensions based on memories of these objects. In this manner, comparable measures of accuracy of spatial memory can be obtained that will permit direct comparisons between individuals.

\section{Measurement of Body Image}

Several researchers have suggested that body image distortion may reflect a more general perceptual distortion (e.g., Auchus, Kose, \& Allen, 1993). Several techniques for assessing body image have already been developed, but most of these have questionable validity (e.g., projective measures), utilize presentations of variations in body morphology that do not capture the actual proportions of most individuals, or have inherently poor precision because they are based on questionnaires (see Cash \& Brown, 1987; Schlundt \& Johnson, 1990). Using the linear estimates indicated by the laser, these judgments may be compared with actual measurements, to obtain an accurate measure of body image distortion for one or more linear dimensions, such as width of the waist, hips, or thighs (see Alley \& Olson, 1997, and above). Likewise, participants may be asked to indicate the "ideal" size for various body parts in order to provide data that can be used to measure the discrepancy between perceived and ideal body size - data that may help predict the potential for eating disorders (Slade, 1985; Zellner, Harner, \& Adler, 1989).

\section{REFERENCES}

Alley, T. R., \& Olson, A. M. (1997). "Body-image" distortion and accuracy of size perception for inanimate objects. In M. A. Schmuckler \& J. M. Kennedy (Eds.), Studies in perception and action IV (pp. 1922). Mahwah, NJ: Erlbaum.

AsKEVold, R. (1975). Measuring body image: Preliminary report on a new method. Psychotherapy \& Psychosomatics, 26, 71-77.

Auchus, M., Kose, G., \& Allen, R. (1993). Body-image distortion and mental imagery. Perceptual \& Motor Skills, 77, 719-728.

CASH, T. F., \& BROWN, T. A. (1987). Body image in anorexia nervosa and bulimia nervosa. Behavior Modification, 11, 487-521.

Cutring, J. E., \& Vishton, P. M. (1995). Perceiving layout and knowing distances: The integration, relative potency, and contextual use of different information about depth. In W. Epstein \& S. Rogers (Eds.), Perception of space and motion (pp. 69-117). San Diego: Academic Press.

GILINSKY, A. S. (1955). The effect of attitude upon the perception of size. American Journal of Psychology, 68, 173-192.

JiANG, Y., \& MARK, L. S. (1994). The effect of gap depth on the perception of whether a gap is crossable. Perception \& Psychophysics, 56, 691-700.

Mark, L. S., Jiang, Y., King, S. S., \& PaAsche, J. (1999). The impact of visual exploration on judgments of whether a gap is crossable. Journal of Experimental Psychology: Human Perception \& Performance, 25, 287-295.

MATIN, L., \& LI, W. (1995). Light and dark adaptation of visually perceived eye level controlled by visual pitch. Perception \& Psychophysics, 57, 84-104.

RufF, G. A., \& Barrios, B. A. (1986). Realistic assessment of body image. Behavioral Assessment, 8, 237-251.

Schiffman, H. R. (1996). Sensation and perception (4th ed.). New York: Wiley.

Schlundt, D. G., \& Johnson, W. G. (1990). Eating disorders: Assessment and treatment. Needham Heights, MA: Allyn \& Bacon.

SEDGWICK, H. A. (1986). Space perception. In K. R. Boff, L. Kaufman, \& J. P. Thomas (Eds.), Handbook of perception and performance: Sensory processes and perception (pp. 21-1 to 21-57). New York: Wiley.

Slade, P. (1985). A review of body-image studies in anorexia nervosa and bulimia nervosa. Journal of Psychiatric Research, 19, 255-265.

Thompson, J. K., \& SPANA, R. E. (1988). The adjustable light beam method for the assessment of size estimation accuracy: Description, psychometric, and normative data. International Journal of Eating Disorders, 7, 521-526.

WARREN, W. H., \& WHANG, S. (1987). Visual guidance of walking through apertures: Body-scaled information for affordances. Journal of Experimental Psychology: Human Perception \& Performance, 13, 371-383.

Zellner, D. A., Harner, D. E., \& Adler, R. L. (1989). Effects of eating abnormalities and gender on perceptions of desirable body shape. Journal of Abnormal Psychology, 98, 93-96.

(Manuscript received March 23, 1999 . revision accepted for publication September 16, 1999.) 